\title{
Mechanisms of Lateral Inhibition in the Olfactory Bulb: Efficiency and Modulation of Spike-Evoked Calcium Influx into Granule Cells
}

\author{
Veronica Egger, Karel Svoboda, and Zachary F. Mainen \\ Cold Spring Harbor Laboratory, Cold Spring Harbor, New York 11724
}

\begin{abstract}
Granule cells are axonless local interneurons that mediate lateral inhibitory interactions between the principal neurons of the olfactory bulb via dendrodendritic reciprocal synapses. This unusual arrangement may give rise to functional properties different from conventional lateral inhibition. Although granule cells spike, little is known about the role of the action potential with respect to their synaptic output. To investigate the signals that underlie dendritic release in these cells, two-photon microscopy in rat brain slices was used to image calcium transients in granule cell dendrites and spines. Action potentials evoked calcium transients throughout the dendrites, with amplitudes increasing with distance from soma and attaining a plateau level within the external plexiform layer, the zone of granule cell synaptic output. Transient amplitudes were, on average, equal in size in spines and adjacent dendrites. Surprisingly, both spine and dendritic amplitudes were strongly dependent on membrane potential, decreasing with depolarization and increasing with hyperpolarization from rest. Both the current-voltage relationship and the time course of inactivation were consistent with the known properties of T-type calcium channels, and the voltage dependence was blocked by application of the T-type calcium channel antagonists $\mathrm{Ni}^{2+}$ and mibefradil. In addition, mibefradil reduced action potential-mediated synaptic transmission from granule to mitral cells. The implication of a transiently inactivating calcium channel in synaptic release from granule cells suggests novel mechanisms for the regulation of lateral inhibition in the olfactory bulb.
\end{abstract}

Key words: olfactory bulb; granule cell; lateral inhibition; action potential; T-type calcium channels; calcium imaging

\section{Introduction}

Granule cells (GCs) are axonless inhibitory interneurons that constitute the majority of neurons in the vertebrate olfactory bulb $(\mathrm{OB})$. They provide the main source of interaction between the principal excitatory neurons of the bulb, the mitral and tufted cells (M/TCs) (Shepherd and Greer, 1998). GCs are central to major aspects of $\mathrm{OB}$ function, yet their role within olfactory processing is still poorly understood. First, they may provide a "spatial" contrast mechanism that sharpens the tuning of M/TC odorant receptive fields, analogous to the role of lateral inhibition in the visual system (Yokoi et al., 1995; Urban, 2002) (but see Laurent, 1999). Next, the reciprocal M/TC-GC synapse may be a site for olfactory plasticity (Kendrick et al., 1992; Wilson and Sullivan, 1994; Hendin et al., 1997). GCs have been implicated in the generation of $\mathrm{OB}$ oscillations and synchrony of M/TC firing (Buonviso et al., 1996; MacLeod and Laurent, 1996; Desmaisons et al., 1999), possibly relevant for odor discrimination (Stopfer et

Received March 25, 2003; revised June 25, 2003; accepted July 3, 2003.

This work was supported by the Deutsche Forschungsgemeinschaft (V.E.), the Burroughs Wellcome Fund (Z.F.M.), the Howard Hughes Medical Institute, and the National Institutes of Health (K.S.). We thank P. O'Brien and B. Burbach for technical help, Dr. B. Sabatini and T. Pologruto for programming custom software, Drs. N. Urban, T. Oertner, N. Uchida, A. Kepecs, R. Malinow, and J. Huguenard for comments on previous versions of this manuscript, and P. Weber and Dr. E. Gutknecht (Hoffmann-La Roche, Basel, Switzerland) for the gift of mibefradil.

Correspondence should be addressed to Dr. Veronica Egger, Cold Spring Harbor Laboratory, Marks Building, 1 Bungtown Road, Cold Spring Harbor, NY 11724. E-mail egger@cshl.edu.

Copyright $\odot 2003$ Society for Neuroscience $\quad$ 0270-6474/03/237551-08\$15.00/0 al., 1997). Finally, GCs receive the majority of cortical feedback to the bulb (Price and Powell, 1970c).

GCs interact with M/TCs via reciprocal dendrodendritic synapses. On the GC, both presynaptic and postsynaptic specializations are found in large spines. This unusual arrangement gives rise to three different GC output modes: (1) Self-inhibition: a single $\mathrm{M} / \mathrm{TC}$ activates a GC spine, which in turn releases GABA back onto the same M/TC (Jahr and Nicoll, 1980, 1982; Isaacson and Strowbridge, 1998). (2) Local lateral inhibition: MT/Cs activate one or more spines in a local region of a GC. Subthreshold activity spreads between spines to cause mutual lateral inhibition between M/TCs (Jahr and Nicoll, 1982; Woolf et al., 1991b; Isaacson and Strowbridge, 1998). (3) Global lateral inhibition: Several M/TCs activate a GC strongly enough to elicit an action potential (AP). Presumably, this AP propagates through the dendritic tree, causing widespread lateral inhibition (Chen et al., 2000).

Little is known about the role of the AP in GC signaling. Robust self-inhibition can be produced without GC APs (Jahr and Nicoll, 1980, 1982), yet in vivo recordings demonstrate that GCs do indeed spike in response to odorants (Mori and Takagi, 1977; Wellis and Scott, 1990; Luo and Katz, 2001; Margrie and Schaefer, 2003; Cang and Isaacson, 2003), and APs can evoke calcium transients in GCs (Hall and Delaney, 2002). Most experiments studying $\mathrm{OB}$ dendrodendritic inhibition have investigated selfinhibition in the presence of TTX (Isaacson and Strowbridge, 1998; Schoppa et al., 1998; Chen et al., 2000; Halabisky et al., 
2000; Isaacson, 2001), and none has isolated GC AP-mediated lateral inhibition. The relative predominance of AP-independent (local) lateral inhibition and AP-dependent (possibly global) lateral inhibition will depend on several factors, including the efficacy of dendritic AP propagation, the types of voltage-dependent calcium channels within spines, and modulatory mechanisms.

Calcium influx into GC dendrites is both an indicator of the spread of neuronal activity and tightly coupled to GC output. Thus, we chose to image AP-evoked GC calcium dynamics at the level of individual spines, using two-photon microscopy.

\section{Materials and Methods}

Preparation, solutions, and instrumentation. In all experiments, we prepared sagittal olfactory bulb brain slices (thickness $350 \mu \mathrm{m}$ ) of juvenile Sprague Dawley rats [postnatal day 10 (P10)-P16]. The preparation was performed in accordance with Cold Spring Harbor Laboratory guidelines for animal care. The intracellular solution contained (in $\mathrm{mM}$ ): 130 K-methylsulfate, 10 HEPES, $4 \mathrm{MgCl}_{2}, 4 \mathrm{Na}_{2} \mathrm{ATP}, 0.4 \mathrm{NaGTP}, 10 \mathrm{Na}$ phosphocreatine, and 2 ascorbate, $\mathrm{pH}$ 7.2. For imaging, $100 \mu \mathrm{M}$ of the calcium-sensitive dye OGB-1 (Molecular Probes, Eugene, OR) was added. The extracellular ACSF was bubbled with carbogen and contained [mM]: $127 \mathrm{NaCl}, 25 \mathrm{NaHCO}_{3}, 1.25 \mathrm{NaH}_{2} \mathrm{PO}_{4}, 25$ glucose, $2.5 \mathrm{KCl}, 1$ $\mathrm{MgCl}_{2}$, and $2 \mathrm{CaCl}_{2}$. The junction potential was $-5 \mathrm{mV}$. Pharmacological agents used in some experiments were APV, CNQX, bicuculline (all from Tocris Cookson, Bristol, UK), TTX (Sigma, St. Louis, MO), and mibefradil (gift from Hoffman-La Roche, Basel, Switzerland). When applying TTX, we increased current injection to obtain the same somatic AP waveform as in control conditions. All experiments were performed at room temperature $\left(21^{\circ} \mathrm{C}\right)$, unless stated otherwise.

Neurons were visualized with infrared differential interference contrast optics. Two-photon excitation at $810 \mathrm{~nm}$ was provided by a Ti: Sapphire solid-state laser system (Mira/Verdi; Coherent, Santa Clara, CA). For a more detailed description of the custom-built two-photon microscope, see Mainen et al. (1999). Somatic whole-cell patch-clamp recordings were performed with an Axopatch 200B (Axon Instruments, Foster City, CA). The pipette resistance was $R_{\mathrm{P}}=5-8 \mathrm{M} \Omega$, and the series resistance was $R_{\mathrm{S}}=15-40 \mathrm{M} \Omega$. APs were elicited by a short current injection in current-clamp mode from resting potential, i.e., $-70 \mathrm{mV}$, unless stated otherwise. After a filling time of $\sim 10 \mathrm{~min}$, calcium transients were imaged in line-scan mode at different locations along the apical dendrite of granule cells and within its spines (Fig. 1A,B). The small size of granule cells promoted fast filling, but also required quick experimentation because cells tended to deteriorate rather abruptly after $25-45 \mathrm{~min}$.

For the experiments on synaptic transmission between granule cells and mitral cells, glass electrodes filled with $\operatorname{ACSF}\left(R_{\mathrm{P}} \approx 1-4 \mathrm{M} \Omega\right)$ and connected to an Isoflex stimulator (A.M.P.I., Jerusalem, Israel) were placed near granule cell somata. Stimulation strengths just sufficient for AP generation in granule cells were determined by test experiments in which granule cells were patched in the whole-cell mode and then extracellularly stimulated.

Data analysis. Imaging and electrophysiological data were recorded and analyzed with custom software based on Matlab (Mathworks, Natick, MA; Pologruto et al., 2003) and Igor (Wavemetrics, Lake Oswego, OR). To measure changes in calcium, fluorescence was collected while scanning in a line that intersected the region or regions of interest. Fluorescence, $F(t)$, was then averaged over the region or regions of interest. Baseline fluorescence, $F_{0}$, was measured for $50 \mathrm{msec}$ before the stimulus, and $\Delta F / F$ was calculated as $(\Delta F / F)(t)=\left(F(t)-F_{0}\right) / F_{0} .(\Delta F / F)_{\mathrm{AP}}$ corresponds to the fluorescence transient evoked by stimulation with a single AP.

For the collective representation of cells, data of individual experiments were normalized to their transient amplitude at the level of the mitral cell layer (MCL). If there was no data point close to the interception of dendrite and MCL, the respective transient amplitude was interpolated. Only neurons with at least three amplitude data points along the apical dendrite were included in the analysis.

To describe voltage dependence of calcium transients, we used two

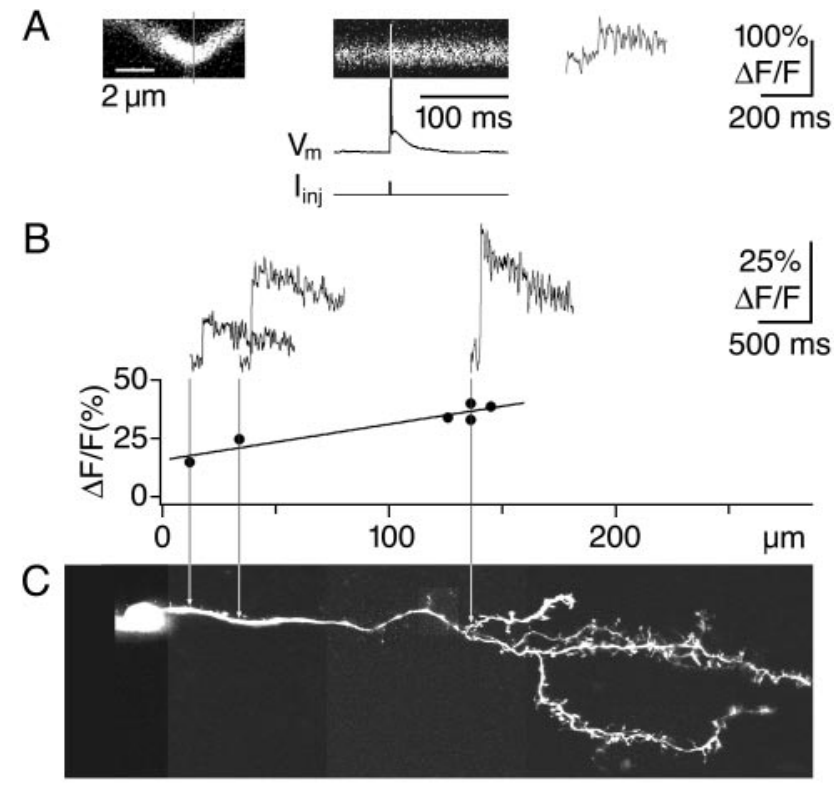

Figure 1. Action potentials produce calcium transients in granule cell dendrites. $A$, Experimental design. The left panel shows a dendrite of the cell in $C$, with the line scan position indicated by the gray vertical line. The middle panel shows the line scan across the dendrite (top), the voltage trace of the corresponding action potential (middle; white/black) evoked by current injection (bottom) to the granule cell soma. The right panel shows the fluorescence transient resulting from this line scan. $B,(\Delta F / F)_{\mathrm{AP}}$ amplitudes do not decrease with distance from the soma. The top traces represent averaged calcium transients imaged at increasing distance from the soma in the cell shown in $C$. The bottom graph shows dendritic $(\Delta F / F)_{A P}$ amplitudes versus distance from the soma and their linear fit. C, Scan of the corresponding granule cell at the same scale. Arrows indicate the measurement locations.

different measures. Data with sufficiently strong voltage dependence and little noise in the individual fluorescence transient amplitudes were fitted with a Boltzmann function. Because this type of fit is not meaningful if applied to data with little voltage dependence or high levels of noise, it was not applicable to the whole population and also not useful to determine block of voltage dependence. Therefore, we evaluated voltage dependence for all measurements in terms of the slope of a linear fit to the data. In any case, based on our Boltzmann analysis yielding an average $V \approx-76 \mathrm{mV}$ and $k \approx 10 \mathrm{mV}$, a linear fit is a fairly good approximation of the data in the range of -90 to $-60 \mathrm{mV}$.

To assess statistical significance levels, the nonparametric Wilcoxon matched-pairs signed-ranks test was applied for comparing data sets, and the Spearman correlation coefficient for establishing correlations. All data are given $\pm \mathrm{SD}$, unless stated otherwise.

\section{Results}

Whole-cell recordings were made in horizontal slices of the olfactory bulb from small cells $(\sim 8 \mu \mathrm{m}$ diameter soma) in the granule cell layer. Because cells were filled with calcium-sensitive fluorescent dye via the pipette, we could identify GCs by the presence of an apical dendrite ascending into the external plexiform layer (EPL) studded with large dendritic spines, also called "gemmules," and a brush of shorter basal dendrites, but lack of an axon (Fig. 1C) (Valverde, 1965; Price and Powell, 1970a).

On average, GC input resistance was $0.81 \pm 0.35 \mathrm{G} \Omega$ (mean \pm SD; $n=72$; Schoppa et al., 1998), the membrane time constant was $30 \pm 14 \mathrm{msec}(n=15)$, and the resting membrane potential was $-69.3 \pm 5.2 \mathrm{mV}(n=32)$, similar to previous in vitro and in vivo data (Wellis and Scott, 1990; Schoppa et al., 1998; Margrie and Schaefer, 2003). Brief, depolarizing current pulses (3 msec; 30-60 pA) elicited single spikes followed by large afterdepolarizations (ADPs) (Fig. 1A). Longer current steps produced char- 
acteristic late-onset firing at low current intensities and a "plateau" pattern at higher intensities, probably reflecting $I_{\mathrm{A}}$ and $I_{\text {CAN }}$, respectively (Schoppa and Westbrook, 1999; Hall and Delaney, 2002). We very rarely observed bursting or rebound APs from hyperpolarizing current steps.

\section{Action potential-evoked dendritic calcium transients}

Fluorescence was measured using line scans across dendrites and spines (Fig. 1A). Single spikes evoked detectable fluorescence transients, $(\Delta F / F)_{\mathrm{AP}}$, in almost all cell locations imaged (367 dendrites and 158 spines in 102 cells). Long trains of $20 \mathrm{APs}$ at $50 \mathrm{~Hz}$ resulted in plateau calcium levels $3.5 \pm 1.6$ times the amplitude of $(\Delta F / F)_{\mathrm{AP}}$ (range, $1.5-6.8 ; n=30$ in 13 cells), indicating that single APs did not saturate the indicator (100 $\mu \mathrm{M}$ OGB-1). Dendritic calcium transients required $\mathrm{Na}^{+}$-dependent APs, as they were blocked by bath application of TTX ( $1 \mu \mathrm{M} ; n=8$ locations in seven cells; see Materials and Methods). The decay of dendritic calcium transients was slow $(\tau=780 \pm 380 \mathrm{msec} ; n=145$ locations). Although the kinetics became faster at physiological temperature $\left(T=34-36^{\circ} \mathrm{C} ; \tau=410 \pm 120 \mathrm{msec}, n=15 ; p<0.005\right.$ for paired experiments, $n=10$; Wilcoxon signed-ranks test for all comparisons), these values are still relatively high compared with transients in pyramidal neurons recorded under similar buffering conditions ( $\tau \approx 400 \mathrm{msec}$ at physiological temperature vs $\tau \approx$ 100 msec: neocortical L5, $100 \mu \mathrm{M}$ CG-1, Markram et al., 1995; $\tau \approx 200$ msec: CA1, $100 \mu \mathrm{M}$ OGB-1, Sabatini et al., 2002). Possible mechanisms for such slow kinetics include a large endogenous buffer capacity, a slow calcium extrusion rate, and calciuminduced calcium release.

Action potentials produce robust dendritic calcium transients We quantified the amplitudes of dendritic calcium transients along the apical dendrite of each GC (Fig. 1). In contrast to observations in pyramidal neurons (see Discussion), these $(\Delta F /$ $F)_{\text {AP }}$ amplitudes did not decrease with distance from the soma (Fig. $1 B$ ). We examined the amplitude of dendritic $(\Delta F / F)_{\mathrm{AP}}$ at different positions along individual dendrites with respect to the border of the EPL, as demarcated by the mitral cell layer. This analysis revealed that an amplitude plateau was often reached at the beginning of the EPL, as depicted in Figure $2 A$. This observation was consistent across the GC population, resulting in a characteristic profile of the relative $(\Delta F / F)_{\mathrm{AP}}$ amplitude with respect to the EPL shown in Figure $2 B(n=98$ cells; see Materials and Methods). A systematic gradient in indicator concentration caused by incomplete loading could produce apparent amplification of transients with distance from the soma, but such a gradient would not be expected to produce a plateau in $(\Delta F / F)_{\mathrm{AP}}$ amplitude. Moreover, $(\Delta F / F)_{\mathrm{AP}}$ decay time constants did not decrease with distance (Fig. $2 C$ ) as would occur if transients were subject to inhomogeneous buffering by the indicator (Neher and Augustine, 1992; Helmchen et al., 1996).

Therefore, dendritic calcium transients provide robust and fairly uniform effects throughout the EPL, where the reciprocal spines are located.

\section{Spine calcium transients}

Transmitter release from granule cells is thought to occur exclusively from dendritic spines within the EPL (Price and Powell, 1970b; Woolf et al., 1991a). Spines and dendrites could show different calcium transient properties caused by differential clearance, buffering, or distribution of calcium channel types. We therefore imaged calcium transients in spines and compared them with the parent dendritic shaft using simultaneous line
A
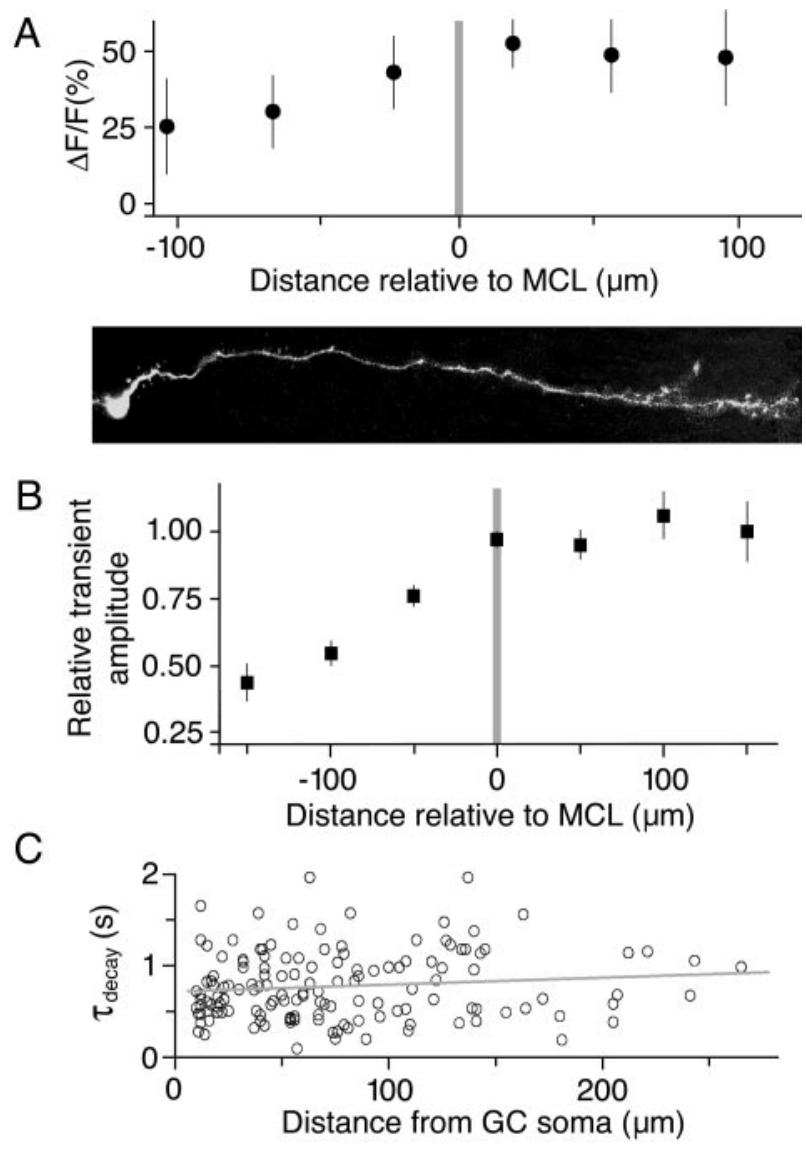

Figure 2. Calcium transients are robust in the external plexiform layer. $A$, Calcium transient amplitudes attain a plateau in the EPL. The top graph represents dendritic $(\Delta F / F)_{A P}$ amplitude data of the individual cell shown below at the same scale versus relative distance to the MCL. The soma of this cell was located at $120 \mu \mathrm{m}$ below the MCL. Amplitude data are shown with SD. $B$, Normalized $(\Delta F / F)_{A P}$ amplitudes averaged over all cells also attain a plateau in the EPL. The graph represents the average amplitudes of all granule cells with at least three dendritic data points ( $n=98$ cells) binned into $50 \mu \mathrm{m}$ intervals, again versus their relative distance to the MCL. Before averaging, data from individual cells were normalized to the amplitude at the MCL border. Data are shown with SEM. $C$, The decay time constant of $(\Delta F / F)_{A P}$ does not depend on distance from the soma. Dendritic data $(n=145)$ with linear fit $(r=0.11)$ is shown. Similar results were obtained for spine measurements ( $r=0.03 ; n=63$; data not shown).

scans through both structures (Fig. 3A). Throughout the EPL, transients were as robust in spines as in dendrites. The average ratio of spine to dendrite $(\Delta F / F)_{\mathrm{AP}}$ amplitude was close to unity: $1.08 \pm 0.49$ (Fig. $3 B)(n=152$ pairs of spine and adjacent dendrite; $p>0.5$ ). In response to AP trains, spines and dendrites also showed a similar transient amplitude, with the dendritic magnitude slightly larger (ratio S/D $0.90 \pm 0.14 ; n=21 ; p<0.01$ ). However, the decay of $(\Delta F / F)_{\mathrm{AP}}$ was significantly faster in spines than in adjacent dendrites (Fig. 3C) $(\tau=640 \pm 50 \mathrm{msec}$ vs $\tau=$ $750 \pm 50$ msec; SEM; $n=52 ; p<0.002)$, indicating that the similarity in $(\Delta F / F)_{\mathrm{AP}}$ amplitudes is not simply caused by rapid equilibration of calcium between spine and dendrite.

We occasionally observed spontaneous synaptic transients $(\Delta F / F)_{\text {syn }}$ (Fig. 3D,E) $(n=12$ spines, except for two all within the EPL; $n=27$ events). Such transients were localized to the spine head, coincided with an EPSP (Fig. 3D) and did not invade the parent dendrite (Fig. $3 F)$ [mean $(\Delta F / F)_{\text {syn }}$ amplitude spine vs dendrite $41 \pm 11$ vs $1 \pm 3 \% \Delta F / F ; p<0.001]$. Therefore, as in pyramidal cells (Svoboda et al., 1996), the spine neck provides a substantial barrier to diffusion over the time scale of $100 \mathrm{msec}$. The average rise time of $(\Delta F / F)_{\text {syn }}$ events was considerably longer 
A

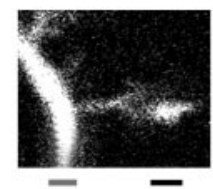

$\mid 2 \mu \mathrm{m}$
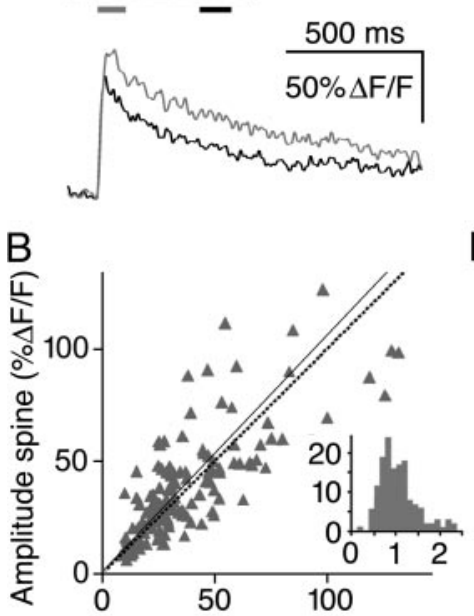

Amplitude dendrite $(\% \Delta \mathrm{F} / \mathrm{F})$

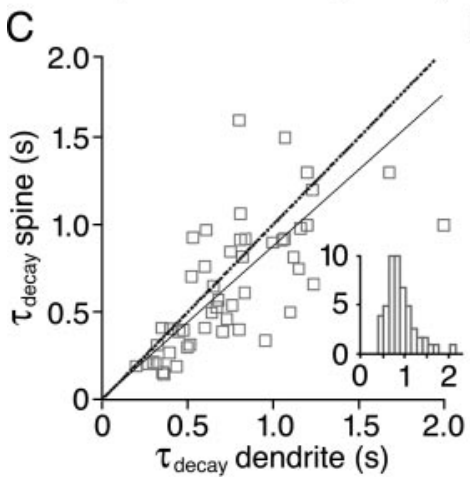

D

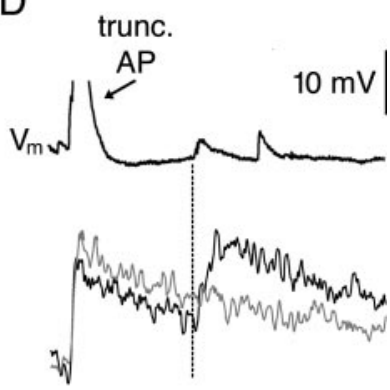

E

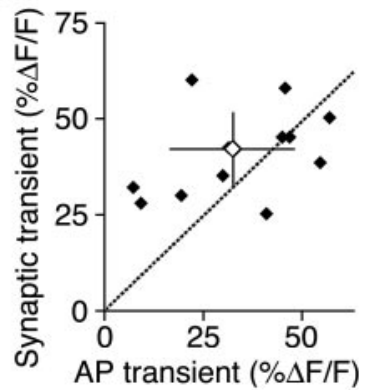

$\mathrm{F}$

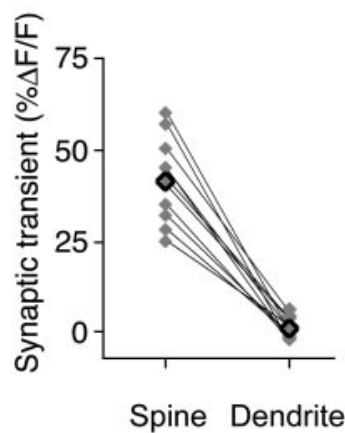

Figure 3. AP and synaptically mediated calcium transients are observed in spines. A, Spine and dendritic $(\Delta F / F)_{A P}$ transients are similar. The scan shows a large spine/gemmule, located at $111 \mu \mathrm{m}$ from the cell soma. Below, the averaged filtered transients in dendrite (gray) and spine (black) are shown, as measured in the regions with respective colors indicated below the scan. The horizontal line scan was aligned with the spine. $B$, Similar $(\Delta F / F)_{A P}$ amplitudes are observed in dendrites and spines. The scatterplot shows transient amplitudes in spines versus transient amplitudes in the adjacent dendrite. The dotted line represents the diagonal $x=y$, and the straight line a linear fit to the data. The inset shows a histogram of amplitude ratios spine/dendrite. C, Slightly faster $(\Delta F / F)_{A P}$ decay is seen in spines than in dendrites. The scatterplot shows transient decay constants in spines versus transient decay time constants in the adjacent dendrite, with details similar to $B$. D, Spontaneous synaptic events occur. The synaptic transient shown was measured in the spine from $A$, with identical scaling. The top trace shows the voltage recording with truncated evoked AP and spontaneous EPSPs. The bottom shows corresponding calcium signals in the spine (black) and adjacent dendrite (gray). Note that the AP evokes a transient both in spine and dendrite, whereas the spontaneous transient is localized to the spine and coincides with a spontaneous EPSP. E, Synaptic and AP-evoked $\Delta F / F$ amplitudes are similar. The scatterplot shows mean synaptic versus AP-evoked $\Delta F / F$ amplitudes in each spine where spontaneous synaptic events were observed $(n=12)$. The open diamond represents the population mean $\pm S D$. F, Synaptic calcium is not observed in the adjacent dendrite. The plot shows mean synaptic $\Delta F / F$ amplitudes in all spine/dendrite pairs where spontaneous synaptic events were observed $(n=12)$. Mean values are represented by open diamonds.

than that of $(\Delta F / F)_{\mathrm{AP}}(70 \pm 37 \mathrm{msec}$ vs $16 \pm 6 \mathrm{msec} ; n=12 ; p<$ $0.005)$, whereas the mean $(\Delta F / F)_{\mathrm{syn}}$ amplitude in spines was similar to that of $(\Delta F / F)_{\mathrm{AP}}$ in the same spines (Fig. 3E) (41 \pm 11 vs $34 \pm 17 \% \Delta F / F ; n=12 ; p>0.25)$. If synaptic and AP-evoked calcium had the same access to the release machinery, this obser-

A

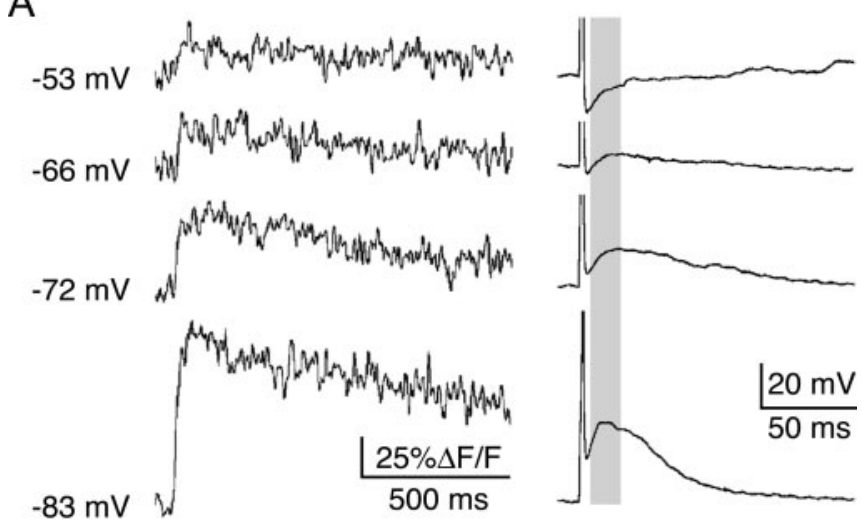

B

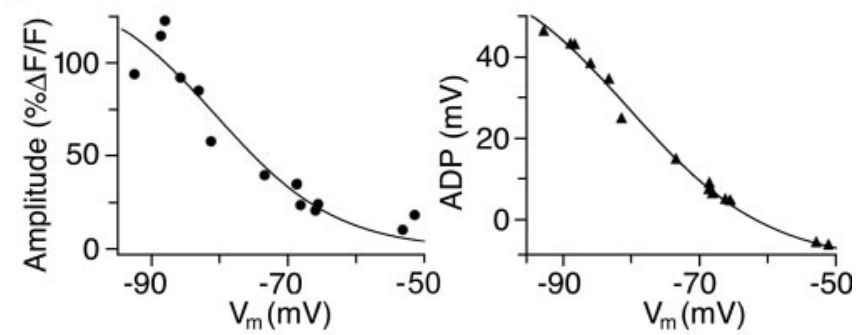

C

D
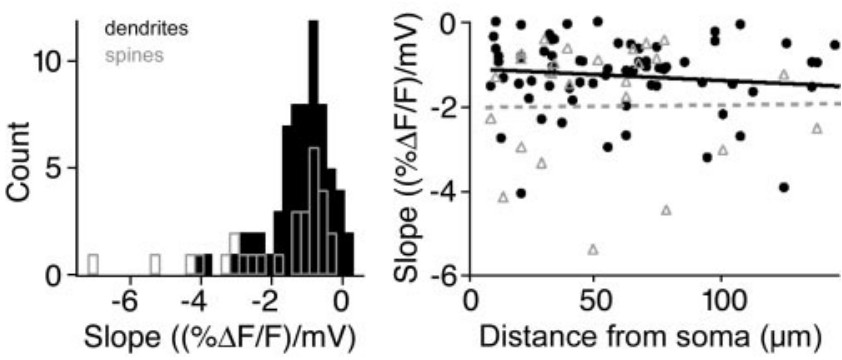

Figure 4. Calcium transient amplitudes are regulated by membrane potential. $A$, Both $(\Delta F)$ $F)_{A P}$ and $A D P$ amplitude are voltage-dependent. The left panel shows representative individual calcium transients at a fixed location ordered at increasingly hyperpolarized pre-pike membrane potentials, the right panel the corresponding APs (truncated) and ADPs. $B$, Voltage dependence obeys a Boltzmann relationship. $(\Delta F / F)_{A P}$ amplitudes (left panel; from individual experiment also shown in $A$ ) and corresponding ADP amplitudes (right panel; calculated as difference between prespike potential and maximum within the shaded interval in $A$ ) are shown as a function of the prespike membrane potential. The Boltzmann fits yield $V_{0.5}=$ $-80.5 \mathrm{mV}, k=8.9 \mathrm{mV}$ for $(\Delta F / F)_{\mathrm{AP}}$ and $V_{0.5}=-80.3 \mathrm{mV}, k=10.2 \mathrm{mV}$ for the ADPs. $C$, Histograms of all locations. The data are represented as histograms of the slopes of linear fits to the transient amplitudes from dendrites ( $n=75$; black solid bars) and spines ( $n=31$; gray open bars). $D$, Voltage dependence is independent on distance from soma. The slopes of linear fits to voltage dependence are shown versus distance of measurement from the soma. Black solid and gray dashed lines are linear fits to dendrite (filled circle) and spine (open triangle) data, respectively.

vation would imply a similar efficiency of the two pathways with respect to causing release and thus inhibition (see Discussion).

\section{Voltage dependence of calcium transients}

Although AP-evoked calcium transients were highly robust, we observed a striking susceptibility of transient amplitudes to the membrane holding potential, being attenuated with depolarization and enhanced with hyperpolarization (Fig. 4A,B, left panels). The average voltage dependence in all dendrites, as described by the linear slope (see Materials and Methods) was $-1.20 \pm 0.86$ $(\% \Delta F / F) / \mathrm{mV}$ ( $n=75$ in 43 neurons) (Fig. $4 C$ ) and stronger for spines than for their adjacent dendrites $[-2.07 \pm 1.71$ vs 
A $-65 \mathrm{mV}$

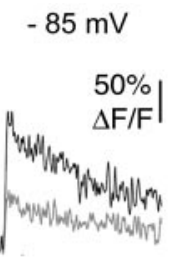

C

Mibefradil
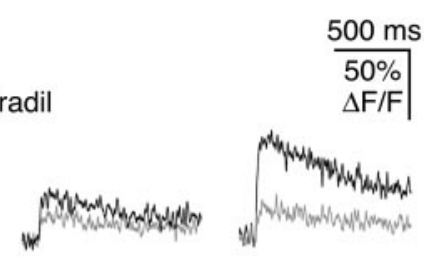

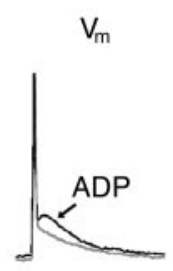

B
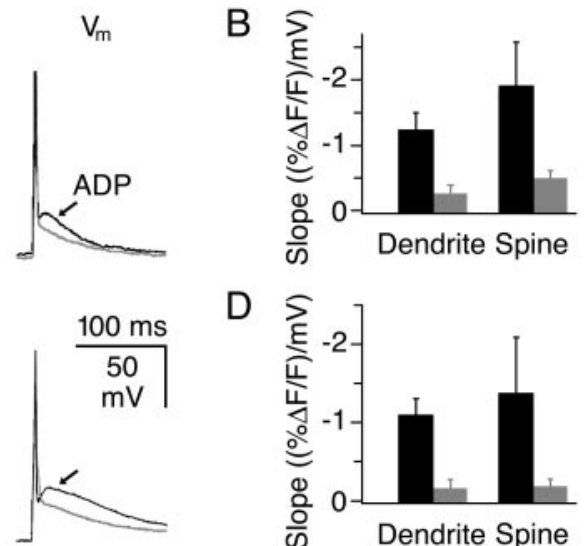

$\mathrm{D}$

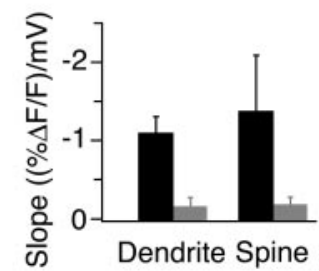

Figure 5. T-type calcium channels underlie voltage dependence of calcium transients. Black traces represent control data and gray traces data with drug applied (100 $\mu \mathrm{m} \mathrm{NiCl}, 1-10 \mu \mathrm{m}$ mibefradil). $A, B, \mathrm{NiCl}_{2}$ reduces $(\Delta F / F)_{A P}$ amplitude voltage dependence and blocks the ADP. C, $D$, Mibefradil also reduces $(\Delta F / F)_{A P}$ amplitude voltage dependence and blocks the ADP. The three panels in $A$ and (show individual experiments. The traces were recorded at prespike potentials of -65 and $-85 \mathrm{mV}$, respectively, and the $A P$ at $-70 \mathrm{mV} . B$ and $D$ show the average linear slope of voltage dependence (see Materials and Methods) in dendrites and spines before and after drug application (error bars indicate SEM; $\mathrm{NiCl}_{2}: n=9$ dendrites, $n=3$ spines; mibefradil: $n=9$ dendrites, $n=5$ spines).

A

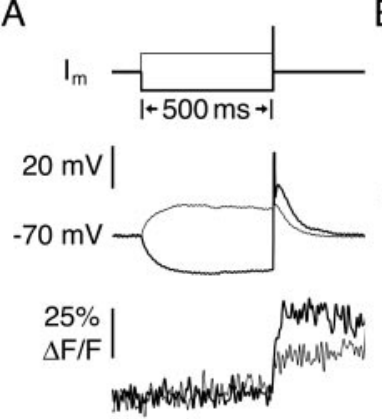

B

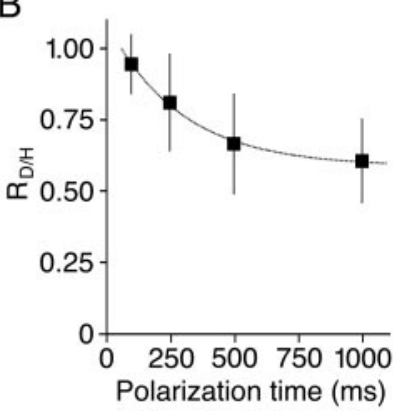

C
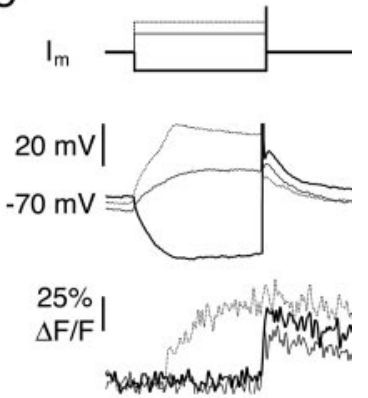

Figure 6. Voltage dependence of calcium transients develops rapidly. In all panels, thick traces mark hyperpolarization to approximately $-90 \mathrm{mV}$, and thin traces depolarization to approximately $-50 \mathrm{mV}$. Before the prepulse, cells were held at approximately $-70 \mathrm{mV}$. $A$, Depolarizing versus hyperpolarizing 500 msec prepulses lead to a pronounced difference in $(\Delta F / F)_{A P}$ amplitudes. The traces from a representative experiment show the injected current (top, schematic), the recorded somatic voltage (middle), and the respective calcium transients (bottom). B, Voltage dependence evolves with a time constant of $\sim 300 \mathrm{msec}$. Cumulative data from all experiments. Average data are plotted as ratios of transient amplitudes for depolarization and hyperpolarization, $R_{\mathrm{D} / \mathrm{H}}$, versus duration of the polarization interval $(100,250,500$, and $1000 \mathrm{msec})$. Data are shown $\pm \mathrm{SD}$. The dotted line corresponds to a single exponential fit ( $\tau=290 \mathrm{msec}$ ). C, Close-to-spiking-threshold depolarization results in considerable calcium influx. Dashed traces mark strong depolarization. Again, the polarization interval was $500 \mathrm{msec}$. Note the characteristic hump in the dashed voltage recording.

$-1.26 \pm 1.04(\% \Delta F / F) / \mathrm{mV} ; n=27$ in 19 neurons; $p<0.01]$. The voltage dependence of $(\Delta F / F)_{\mathrm{AP}}$ amplitudes was not caused by changes in the width or peak amplitude of the somatic AP, because both increased slightly with depolarization $(n=5)$. Furthermore, voltage dependence was not dependent on the distance of the measurement location from the soma $(r=-0.12 /-0.03$ for dendrites/spines; $n=67 / 27$ ) (Fig. $4 D$ ). Spike ADP amplitude was inversely dependent on the membrane potential in a manner closely paralleling the effect on calcium transients (Fig. $4 A$, right panel), suggesting a calcium dependence of the conductance underlying the ADP.

The voltage dependence of both ADP and, in locations with sufficiently large voltage dependence, $(\Delta F / F)_{\mathrm{AP}}$, could be fitted with Boltzmann functions (Fig. $4 B$ ). This fit yielded a mean halfinactivation voltage $V_{0.5}=-76.1 \pm 7.4 \mathrm{mV}$ and a Boltzmann slope factor, $k=9.8 \pm 5.1 \mathrm{mV}$ for calcium transients $(n=25$ locations; $n=13$ cells), and $V_{0.5}=-78.1 \pm 5.3 \mathrm{mV}$ and $k=$
$8.1 \pm 1.9 \mathrm{mV}$ for ADPs in the same 13 cells. This voltage dependence is similar to the characteristic profile for inactivation of low voltage-activated (LVA) or T-type calcium channels (T-channels; Huguenard, 1996).

Role of low voltage-activated channels in regulation of calcium transients

LVA calcium currents have not been previously described in GCs, but the calcium channel $\alpha$ subunits that encode T-channels are known to be expressed richly in these cells (Talley et al., 1999). We tested the effect of two T-channel antagonists. Application of $\mathrm{Ni}^{2+}(100 \mu \mathrm{M})$ caused a significant reduction in both the amplitudes (to $50 \pm 16 \%$ of control) and the voltage dependence of calcium transients (Fig. 5A,B) [control, $-1.68 \pm 0.91$; drug, $-0.29 \pm 0.41(\% \Delta F / F) / \mathrm{mV} ; n=12$ locations in 6 cells; $p<0.002$ for both; dendrites and spines pooled]. Dendrite and spine data are shown separately in Figure $5, B$ and $D$. Similarly, the more specific, activity-dependent $\mathrm{T}$-channel blocker mibefradil $(1-10 \mu \mathrm{M}$; Bezprozvanny and Tsien, 1995; Lacinova et al., 2000) reduced both $(\Delta F / F)_{\mathrm{AP}}$ amplitudes $(49 \pm 12 \%$ of control) and their voltage dependence (Fig. $5 C, D$ ) [control, $-1.20 \pm 1.00$; drug, $-0.29 \pm 0.28(\% \Delta F / F) / \mathrm{mV} ; n=14$ locations in five cells; $p<0.001$ for both]. The high concentrations of the T-channel blockers used and the negligible voltage dependence of the remaining 50\% of the calcium signal imply that this remaining signal was not carried by LVA calcium channels. Both blockers also abolished or reduced the ADP (Fig. 5A,C) without affecting the rise time of calcium transients $(n=10)$. Given the slow time course of the ADP (decay time constant $\tau=80 \pm 50$ msec; $\left.n=15 ; V_{\mathrm{m}}=-70 \mathrm{mV}\right)$, the lack of sensitivity of $(\Delta F / F)_{\mathrm{AP}}$ rise time to $\mathrm{ADP}$ blockade indicates that the ADP reflects a $\mathrm{Ca}^{2+}$-activated current rather than the T-current itself. Finally, mibefradil had no effect on GC spiking (data not shown): it did not change the amount of current required to elicit single APs $(n=5)$, or the number of spikes in response to 500 msec depolarizing current steps $(n=3)$ or oscillatory current injections $(n=5)$.

\section{Time course of calcium transient modulation}

To investigate the time course of calcium transient modulation by voltage, we applied a timed $20 \mathrm{mV}$ depolarizing or hyperpolarizing prepulse to inactivate or deinactivate LVA calcium channels (Fig. 6A) (Magee et al., 1995). We varied the duration of the prepulse and calculated the ratio of $(\Delta F / F)_{\mathrm{AP}}$ amplitudes at depolarized and hyperpolarized potentials, $R_{\mathrm{D} / \mathrm{H}}$ (Fig. $6 B$ ). After $500 \mathrm{msec}, R_{\mathrm{D} / \mathrm{H}}$ was $0.66 \pm 0.18(n=32)$ and almost saturated, corresponding to a time constant of $\sim 290 \mathrm{msec}$. This time course is consistent with reported time constants for T-channel inacti- 
vation and deinactivation at these membrane potentials (Huguenard, 1996; Randall and Tsien, 1997; Lacinova et al., 2000).

Larger amplitude depolarizing pulses to just below AP threshold (mean depolarization, $-40.9 \pm 6 \mathrm{mV}$ ) resulted in substantial calcium influx $(\Delta F / F)_{-40 \mathrm{mV}}$ (Fig. $6 C)$. The average $(\Delta F / F)_{-40 \mathrm{mV}}$ level achieved with $500 \mathrm{msec}$ of depolarization was $42.3 \pm 20.6 \% \Delta F / F(n=23)$. This activation voltage is consistent with T-type calcium channels (Randall and Tsien, 1997). Indeed, the $(\Delta F / F)_{-40 \mathrm{mV}}$ amplitude was reduced substantially by $10 \mu \mathrm{M}$ mibefradil $(27 \pm 10 \%$ of control; $n=9 ; p<$ 0.005). These observations also indicate that subthreshold depolarization can produce calcium influx through T-channels that may lead to local lateral inhibition (see Discussion).

\section{Role of T-type calcium channels in synaptic release}

Do T-type calcium channels participate in AP-mediated transmitter release from granule cells? Their involvement seems likely, given our finding that $\mathrm{T}$-channel blockers reduced the $(\Delta F / F)_{\mathrm{AP}}$ amplitude in dendrites and spines by $50 \%$. To test this more directly, we first sought to examine the effect of depolarization or hyperpolarization on GC output using paired recordings of granule and mitral cells $(n>100)$. However, this approach was precluded by an extremely low success rate in finding connected pairs (cf. Isaacson, 2001). We therefore evoked APs in GCs using extracellular stimulation and recorded in whole-cell mode from mitral cells (Fig. 7A). To prevent triggering of the polysynaptic local lateral inhibition pathway (which does not involve GC spiking) via stimulation of mitral cell axons, we blocked transmission from mitral cells to GCs with APV and CNQX (50 $\mu \mathrm{M} ; 10 \mu \mathrm{M}$; Chen et al., 2000). Under these conditions, small $(0.83 \pm 0.51$ $\mathrm{mV} ; n=6)$, short-latency IPSPs with a slow decay time constant $(\tau=300 \pm 200 \mathrm{msec} ; n=6)$ could be evoked in some mitral cells. Mibefradil $(10 \mu \mathrm{M})$ reduced these evoked IPSPs by $48 \pm 6 \%(p<$ 0.025) (Fig. 7B,C). This decrease is unlikely to be caused by a change in stimulation efficiency or input resistance, because neither GC excitability in whole-cell recordings (see Results above) nor the amplitude and kinetics of spontaneous IPSPs $(n=3)$ were affected by mibefradil application. Subsequent application of bicuculline $(50 \mu \mathrm{M})$ abolished the IPSP in all cases tested $(n=$ 3 ), demonstrating that the IPSPs were mediated by GABA-A receptors. Mibefradil-sensitive calcium channels therefore contribute at least half of the effect of global (AP-mediated) lateral inhibition.

\section{Discussion}

Our results indicate that somatically evoked APs cause calcium transients throughout the GC dendritic tree. These calcium transients are particularly robust in the output zone of GCs and thus appear well suited to evoke transmitter release from most or all release sites. Thus, we refer to this GC output mode as "global lateral inhibition" (Chen et al., 2000). We find that AP-evoked calcium transients are subject to voltage-dependent modulation, apparently because of the contribution of T-type calcium channels, giving rise to novel mechanisms for regulation of lateral inhibition.
B

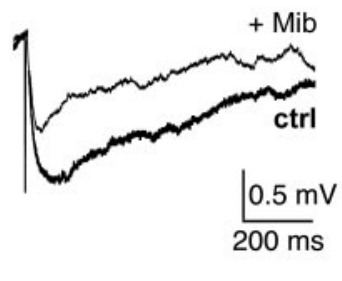

C

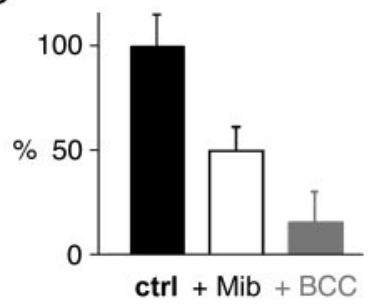

Figure 7. Action potential-mediated synaptic transmission from granule cells to mitral cells is reduced by a T-type calcium channel antagonist. $A$, Experimental stimulation and recording scheme. Granule cells are stimulated extracellularly, whereas a .

\section{Action potential-evoked calcium transients in dendrites}

In contrast to many other cell types studied (e.g., CA1 pyramidal neurons, Spruston et al., 1995; neocortical layer (L) 2/3 pyramidal neurons, Svoboda et al., 1999; L2/3 interneurons, Kaiser et al., 2001) AP-evoked GC calcium transients were robust in even the most distal regions of the dendritic tree imaged and in the large spines in the EPL that are the site of dendritic transmitter release. Data from OB MCs (Xiong and Chen, 2002) (but see Margrie et al., 2001), retinal amacrine cells (Euler et al., 2002), and thalamic GABAergic interneurons (Munsch et al., 1997), are consistent with the idea that upregulation of calcium influx within output regions may be a general rule for cell types with dendritic release. The factors that determine GC dendritic calcium transient amplitudes remain to be elucidated, but could involve spatial gradients in calcium channel distributions (Christie et al., 1995) or effects of passive dendritic electrical properties on AP propagation (Spruston et al., 1995; Vetter et al., 2001).

\section{Voltage-dependent calcium transients mediated by $\mathrm{T}$-channels}

Whereas axonal spikes and bouton calcium transients are essentially all or none (Mackenzie et al., 1996; Cox et al., 2000; Koester and Sakmann, 2000), GC dendritic calcium transients were strongly modulated by membrane potential. Several lines of evidence support the idea that T-type calcium channels underlie this modulation. First, the voltage dependence and its time course were consistent with characteristics of T-channel inactivation (Huguenard, 1996; Lacinova et al., 2000). Second, the R- and T-channel blocker $\mathrm{Ni}^{2+}$ and the more selective $\mathrm{T}$-channel antagonist mibefradil (Bezprozvanny and Tsien, 1995; Lacinova et al., 2000) abolished the voltage dependence and partially blocked the calcium transients. Third, although the T-channel antagonists used may also block high voltage-activated (HVA) calcium channels, in particular R-type (Bezprozvanny and Tsien, 1995; Jimenez et al., 2000), and inactivation relationships of $\mathrm{T}$ - and R-channels may be similar (Randall and Tsien, 1997), subthreshold depolarizing pulses, which will not activate R-channels, caused considerable calcium influx. Finally, GCs express the mRNA transcripts of all three known subtypes of T- channels at high levels ( $\alpha 1 \mathrm{G}, \mathrm{H}$, I; Talley et al., 1999).

The resting potential of GCs $(-70 \mathrm{mV})$ is well suited to allow for T-channel based modulation of calcium dynamics in both directions of polarization. Although we did not observe electrophysiological hallmarks of T-channels such as rebound spikes or bursting, the strong A-type potassium conductance in GCs (Schoppa and Westbrook, 1999) may obscure these effects, as 
described in dendritically releasing thalamic GABAergic interneurons (Pape et al., 1994).

\section{The coupling of calcium to transmitter release}

Release from axonal boutons is triggered by calcium influx via several types of HVA calcium channels (Fisher and Bourque, 2001). In granule reciprocal spines however, calcium entry via NMDA receptors has also been linked to release (Schoppa et al., 1998; Chen et al., 2000; Halabisky et al., 2000) (but see Isaacson, 2001). Our data have added another potential pathway for release, calcium entry via LVA channels, which have only been known to play a role in graded release from retinal bipolar neurons so far (Pan et al., 2001). Because the resolution of our fluorescence measurements does not reveal directly the calcium signal available to the release machinery, the precise nature of this coexistence of pathways remains to be elucidated. The coupling of diverse calcium sources to release could be simply attributable to the proximity of all these sources to the release machinery. It is also conceivable that release from granule cells is sensitive to lower levels of calcium, and hence there is an extended spatial domain for calcium entry from which release may be triggered, allowing for a larger variety of calcium sources to contribute to release (but see Isaacson, 2001). The latter scenario could help to explain the phenomenon of asynchronous release from granule cells (Isaacson and Strowbridge, 1998; Schoppa et al., 1998). Asynchronous release underlies the slow decay of IPSCs and IPSPs generally observed in self- and lateral inhibition and is consistent with the slow time course of IPSPs evoked by extracellular stimulation of GCs. The relatively slow kinetics of GC calcium transients we observed may also contribute to an extended time window for release.

\section{Implications for local and self-inhibition}

Coexisting calcium sources for release are likely to be accessed differentially by APs and synaptic events. Whereas NMDARs would be only available to a spine via direct synaptic input, LVA calcium channels could be recruited both by APs and by synaptic depolarizations, because they do not require APs to reach activating voltages (Magee and Johnston, 1995; Magee et al., 1995). Indeed, subthreshold depolarizing steps were capable of producing robust calcium transients in GC spines. Apparently, in GCs HVA calcium channels can also be activated synaptically (i.e., in TTX: Isaacson, 2001). Consequentially the different GC output modes, self-inhibition, local and global lateral inhibition, would also rely on these calcium sources in a differential manner. For example, self-inhibition appears less susceptible to blockade by $\mathrm{Ni}^{2+}$ (Isaacson and Strowbridge, 1998) than global lateral inhibition. Thus, the relative balance of self-inhibition and lateral inhibition could be specifically regulated.

In addition, our observations suggest that there may be a threshold for local lateral inhibition. Individual spontaneous synaptic transients do not lead to calcium spread into the dendrite or adjacent spines, and subthreshold depolarizations must exceed $\sim 25 \mathrm{mV}$ threshold from resting potential before calcium channels are substantially activated. Thus, spatial or temporal summation of neighboring MC input to a local dendrite would be required to produce local lateral inhibition. In turn, the existence of a threshold for local lateral inhibition suggests the involvement of a regenerative mechanism, perhaps involving voltagedependent calcium channels. Indeed, both full-blown spikes and spikelets of potentially dendritic origin have been recorded in vivo (Mori and Takagi, 1978; Wellis and Scott, 1990; Luo and Katz, 2001).

\section{Consequences of voltage-dependent lateral inhibition}

Because of the voltage dependence of GC calcium influx, subthreshold depolarizing or hyperpolarizing inputs to GCs will modulate lateral inhibition in the $\mathrm{OB}$. One functional consequence of this activity dependence is that lateral inhibition will decrease during periods of prolonged MC firing (Urban and Sakmann, 2002). In vivo, a marked adaptation of GC output was observed during extended odor presentation (Cang and Isaacson, 2003). In addition, GCs are the primary target of centrifugal input to the $\mathrm{OB}$, including excitatory input from olfactory cortex (Price and Powell, 1970b; Nakashima et al., 1978) and inhibitory input from the nucleus of the horizontal limb of the diagonal band (Kunze et al., 1992). The dependence of GC output on membrane potential provides a "gate" by which central feedback could modulate $\mathrm{OB}$ functional connectivity.

Because of the time scale of their activation and inactivation, $\mathrm{T}$-channels are involved in oscillatory activity at slow frequencies in a variety of neurons (Huguenard, 1996). In the OB, respiratory related oscillatory activity at $4-10 \mathrm{~Hz}$ (theta rhythm) is particularly prominent (Macrides and Chorover, 1972; Chaput, 1986; Margrie and Schaefer, 2003), and in vivo studies have linked the frequency and phase of GC spiking with the respiratory cycle (Ravel et al., 1987; Young and Wilson, 1999; Margrie and Schaefer, 2003; Cang and Isaacson, 2003). Our results suggest that subthreshold theta frequency input to granule cells might lead to periodic oscillatory modulation of lateral inhibition in the olfactory bulb.

\section{References}

Bezprozvanny I, Tsien RW (1995) Voltage-dependent blockade of diverse types of voltage-gated $\mathrm{Ca}^{2+}$ channels expressed in Xenopus oocytes by the $\mathrm{Ca}^{2+}$ channel antagonist mibefradil (Ro 40-5967). Mol Pharmacol 48:540-549.

Buonviso N, Chaput MA, Berthommier F (1996) Similarity of granularinduced inhibitory periods in pairs of neighboring mitral/tufted cells. J Neurophysiol 76:2393-2401.

Cang J, Isaacson JS (2003) In vivo whole-cell recording of odor-evoked synaptic transmission in the rat olfactory bulb. J Neurosci 23:4108-4116.

Chaput MA (1986) Respiratory-phase-related coding of olfactory information in the olfactory bulb of awake freely breathing rabbits. Physiol Behav 36:319-324.

Chen WR, Xiong W, Shepherd GM (2000) Analysis of relations between NMDA receptors and GABA release at olfactory bulb reciprocal synapses. Neuron 25:625-633.

Christie BR, Eliot LS, Ito K, Miyakawa H, Johnston D (1995) Different Ca ${ }^{2+}$ channels in soma and dendrites of hippocampal pyramidal neurons mediate spike-induced $\mathrm{Ca}^{2+}$ influx. J Neurophysiol 173:2553-2557.

Cox CL, Denk W, Tank DW, Svoboda K (2000) Action potentials reliably invade axonal arbors of rat neocortical neurons. Proc Natl Acad Sci USA 97:9724-9728.

Desmaisons D, Vincent JD, Lledo PM (1999) Control of action potential timing by intrinsic subthreshold oscillations in olfactory bulb output neurons. J Neurosci 19:10727-10737.

Euler T, Detwiler PB, Denk W (2002) Directionally selective calcium signals in dendrites of starburst amacrine cells. Nature 418:845-852.

Fisher TE, Bourque CW (2001) The function of $\mathrm{Ca}(2+)$ channel subtypes in exocytotic secretion: new perspectives from synaptic and non-synaptic release. Prog Biophys Mol Biol 77:269-303.

Halabisky B, Friedman D, Radojicic M, Strowbridge BW (2000) Calcium influx through NMDA receptors directly evokes GABA release in olfactory bulb granule cells. J Neurosci 20:5124-5134.

Hall BJ, Delaney KR (2002) Contribution of a calcium-activated nonspecific conductance to NMDA receptor-mediated synaptic potentials in granule cells of the frog olfactory bulb. J Physiol (Lond) 543:819-834.

Helmchen F, Imoto K, Sakmann B (1996) $\mathrm{Ca}^{2+}$ buffering and action potential-evoked $\mathrm{Ca}^{2+}$ signaling in dendrites of pyramidal neurons. Biophys J 70:1069-1081.

Hendin O, Horn D, Tsodyks MV (1997) The role of inhibition in an asso- 
ciative memory model of the olfactory bulb. J Comput Neurosci 4:173-182.

Huguenard JR (1996) Low-threshold calcium currents in central nervous system neurons. Annu Rev Physiol 58:329-348.

Isaacson JS (2001) Mechanisms governing dendritic gamma-aminobutyric acid (GABA) release in the rat olfactory bulb. Proc Natl Acad Sci USA 98:337-342.

Isaacson JS, Strowbridge BW (1998) Olfactory reciprocal synapses: dendritic signaling in the CNS. Neuron 20:749-761.

Jahr CE, Nicoll RA (1980) Dendrodendritic inhibition: demonstration with intracellular recording. Science 207:1473-1475.

Jahr CE, Nicoll RA (1982) An intracellular analysis of dendrodendritic inhibition in the turtle in vitro olfactory bulb. J Physiol (Lond) 326:213-234.

Jimenez C, Bourinet E, Leuranguer V, Richard S, Snutch TP, Nargeot J (2000) Determinants of voltage-dependent inactivation affect mibefradil block of calcium channels. Neuropharmacology 39:1-10.

Kaiser KM, Zilberter Y, Sakmann B (2001) Back-propagating action potentials mediate calcium signalling in dendrites of bitufted interneurons in layer 2/3 of rat somatosensory cortex. J Physiol (Lond) 535:17-31.

Kendrick KM, Levy F, Keverne EB (1992) Changes in the sensory processing of olfactory signals induced by birth in sheep. Science 256:833-836.

Koester HJ, Sakmann B (2000) Calcium dynamics associated with action potentials in single nerve terminals of pyramidal cells in layer $2 / 3$ of the young rat neocortex. J Physiol (Lond) 529:625-646.

Kunze WA, Shafton AD, Kem RE, McKenzie JS (1992) Intracellular responses of olfactory bulb granule cells to stimulating the horizontal diagonal band nucleus. Neuroscience 48:363-369.

Lacinova L, Klugbauer N, Hofmann F (2000) Low voltage activated calcium channels: from genes to function. Gen Physiol Biophys 19:121-136.

Laurent G (1999) A systems perspective on early olfactory coding. Science 286:723-728.

Luo M, Katz LC (2001) Response correlation maps of neurons in the mammalian olfactory bulb. Neuron 32:1165-1179.

Mackenzie PJ, Umemiya M, Murphy TH (1996) $\mathrm{Ca}^{2+}$ imaging of CNS axons in culture indicates reliable coupling between single action potentials and distal functional release sites. Neuron 16:783-795.

MacLeod K, Laurent G (1996) Distinct mechanisms for synchronization and temporal patterning of odor-encoding neural assemblies. Science 274:976-979.

Macrides F, Chorover SL (1972) Olfactory bulb units: activity correlated with inhalation cycles and odor quality. Science 175:84-87.

Magee JC, Johnston D (1995) Synaptic activation of voltage-gated channels in the dendrites of hippocampal pyramidal neurons. Science 268:301-304.

Magee JC, Christofi G, Miyakawa H, Christie B, Lasser-Ross N, Johnston D (1995) Subthreshold synaptic activation of voltage-gated $\mathrm{Ca}^{2+}$ channels mediates a localized $\mathrm{Ca}^{2+}$ influx into the dendrites of hippocampal pyramidal neurons. J Neurophysiol 74:1335-1342.

Mainen ZF, Maletic-Savatic M, Shi SH, Hayashi Y, Malinow R, Svoboda K (1999) Two-Photon imaging in living brain slices. Comp Methods Enzymol 18:231-239.

Margrie TW, Schaefer AT (2003) Theta oscillation coupled spike latencies yield computational vigour in a mammalian sensory system. J Physiol (Lond) 546:363-374.

Margrie TW, Sakmann B, Urban NN (2001) Action potential propagation in mitral cell lateral dendrites is decremental and controls recurrent and lateral inhibition in the mammalian olfactory bulb. Proc Natl Acad Sci USA 98:319-324.

Markram H, Helm PJ, Sakmann B (1995) Dendritic calcium transients evoked by single back-propagating action potentials in rat neocortical pyramidal neurons. J Physiol (Lond) 485:1-20.

Mori K, Takagi SF (1977) Inhibition in the olfactory bulb: Dendrodendritic interactions and their relation to the induced waves. In: Food intake and chemical senses (Satoh M, Katsuki Y, Takagi SF, Oomura Y, eds), pp. 33-43. Tokyo: University of Tokyo Press.

Mori K, Takagi SF (1978) Intracellular study of dendrodendritic inhibitory synapses on mitral cells in the rabbit olfactory bulb. J Physiol (Lond) 279:569-588.

Munsch T, Budde T, Pape HC (1997) Voltage-activated intracellular calcium transients in thalamic relay cells and interneurons. NeuroReport 8:2411-2418.

Nakashima M, Mori K, Takagi SF (1978) Centrifugal influence on olfactory bulb activity in the rabbit. Brain Res 154:301-316.
Neher E, Augustine GJ (1992) Calcium gradients and buffers in bovine chromaffin cells. J Physiol (Lond) 450:273-301.

Pan ZH, Hu HJ, Perring P, Andrade R (2001) T-type Ca(2+) channels mediate neurotransmitter release in retinal bipolar cells. Neuron 32:89-98.

Pape HC, Budde T, Mager R, Kisvarday ZF (1994) Prevention of $\mathrm{Ca}^{(2+)}$ mediated action potentials in GABAergic local circuit neurones of rat thalamus by a transient K+ current. J Physiol (Lond) 478:403-422.

Pologruto TA, Sabatini BL, Svoboda K (2003) ScanImage: flexible software for operating laser scanning microscopes. Biomed Eng Online 2:13.

Price JL, Powell TPS (1970a) The morphology of the granule cells of the olfactory bulb. J Cell Sci 7:91-123.

Price JL, Powell TPS (1970b) The synaptology of the granule cells of the olfactory bulb. J Cell Sci 7:125-155.

Price JL, Powell TPS (1970c) An electron-microscopic study of the termination of the afferent fibres to the olfactory bulb from the cerebral hemisphere. J Cell Sci 7:157-187.

Randall AD, Tsien RW (1997) Contrasting biophysical and pharmacological properties of T-type and R-type calcium channels. Neuropharmacology 36:879-893.

Ravel N, Caille D, Pager J (1987) A centrifugal respiratory modulation of olfactory bulb unit activity: a study on acute rat preparation. Exp Brain Res 65:623-628.

Sabatini BL, Oertner TG, Svoboda K (2002) The life cycle of $\mathrm{Ca}^{(2+)}$ ions in dendritic spines. Neuron 33:439-452.

Schoppa NE, Westbrook GL (1999) Regulation of synaptic timing in the olfactory bulb by an A-type potassium current. Nat Neurosci 2:1106-1113.

Schoppa NE, Kinzie JM, Sahara Y, Segerson TP, Westbrook GL (1998) Dendrodendritic inhibition in the olfactory bulb is driven by NMDA receptors. J Neurosci 18:6790-6802.

Shepherd GM, Greer CA (1998) The olfactory bulb. In: The synaptic organization of the brain (Shepherd GM, ed.), pp 133-169. New York: Oxford UP.

Spruston N, Schiller Y, Stuart G, Sakmann B (1995) Activity-dependent action potential invasion and calcium influx into hippocampal CA1 dendrites. Science 268:297-300.

Stopfer M, Bhagavan S, Smith BH, Laurent G (1997) Impaired odour discrimination on desynchronization of odour-encoding neural assemblies. Nature 390:70-74.

Svoboda K, Tank DW, Denk W (1996) Direct measurement of coupling between dendritic spines and shafts. Science 272:716-719.

Svoboda K, Helmchen F, Denk W, Tank DW (1999) Spread of dendritic excitation in layer $2 / 3$ pyramidal neurons in rat barrel cortex in vivo. Nat Neurosci 2:65-73.

Talley EM, Cribbs LL, Lee JH, Daud A, Perez-Reyes E, Bayliss DA (1999) Differential distribution of three members of a gene family encoding low voltage-activated (T-type) calcium channels. J Neurosci 19:1895-1911.

Urban NN (2002) Lateral inhibition in the olfactory bulb and in olfaction. Physiol Behav 6821:1-6.

Urban NN, Sakmann B (2002) Reciprocal intraglomerular excitation and intra- and interglomerular lateral inhibition between mouse olfactory bulb mitral cells. J Physiol (Lond) 542:355-367.

Valverde F (1965) Studies on the piriform lobe. Cambridge: Harvard UP.

Vetter P, Roth A, Hausser M (2001) Propagation of action potentials in dendrites depends on dendritic morphology. J Neurophysiol 85:926-939.

Wellis DP, Scott JW (1990) Intracellular responses of identified rat olfactory bulb interneurons to electrical and odor stimulation. J Neurophysiol 64:932-947.

Wilson DA, Sullivan RM (1994) Neurobiology of associative learning in the neonate: early olfactory learning. Behav Neural Biol 61:1-18.

Woolf TB, Shepherd GM, Greer CA (1991a) Serial reconstructions of granule cell spines in the mammalian olfactory bulb. Synapse 7:181-192.

Woolf TB, Shepherd GM, Greer CA (1991b) Local information processing in dendritic trees: subsets of spines in granule cells of the mammalian olfactory bulb. J Neurosci 11:1837-1854.

Xiong W, Chen WR (2002) Dynamic gating of spike propagation in the mitral cell lateral dendrites. Neuron 34:115-126.

Yokoi M, Mori K, Nakanishi S (1995) Refinement of odor molecule tuning by dendrodendritic synaptic inhibition in the olfactory bulb. Proc Natl Acad Sci USA 92:3371-3375.

Young TA, Wilson DA (1999) Frequency-dependent modulation of inhibition in the rat olfactory bulb. Neurosci Lett 279:65-67. 